\title{
Michael Schuenke, Erik Schulte, Udo Schumacher, Laurence M. Ross, Edward D. Lamperti: THIEME atlas of anatomy image collection general anatomy and musculoskeletal system
}

\author{
Georg Thieme Verlag, Stuttgart, New York, 2007 1.694 illustrations, 100 tables on DVD, \\ US \$ 199.95, ISBN 13: 978-1-58890-489-8 (TNY), ISBN 13: 978-3 131437419 (GTV)
}

\author{
Pierre Kehr
}

Received: 16 August 2009 / Accepted: 18 August 2009 / Published online: 1 September 2009

(C) Springer-Verlag 2009

This is a superb DVD that allows easy and intuitive use. The four headings in the left column are: General anatomy, Trunk wall, Upper limb, and Lower limb.

When you click on GENERAL ANATOMY, a tree view control expands to show eight subheadings, which are divided into a great number of sections, from 2 to 10 depending on the selected item. The user has to move down to the last available section of every chapter to display figures in the large blank area on the right part of the screen. Then, you will be pleasantly surprised at the clarity of the figures and diagrams!

Moreover, each page containing figures include excellent tools. An option list is shown on the upper portion with the following functions: «Back »; « With/without label (allowing not to display labels) »; « Copy to clipboard (enabling us to copy the clipboard content in 72 dpi, especially useful to insert files into a PowerPoint presentation) »; « Save as ... (for 300 dpi enlargements) »; « Zoom (with three levels of magnification) ».

Each one of the chapters: Trunk wall, Upper limb and Lower limb is divided into five sections, which have the same title: « Bones, Ligaments and Joints »; « Musculature: Functional Groups"; "Musculature: Topographical Anatomy" »; « Neurovascular Systems, Forms and Relations"; Neurovascular Systems:Topographical Anatomy ».

You will find a keyword "Search" button at the top of the same left column.
Keywords have to be entered in English and the process can seem puzzling at first: for example, if you enter « femoral artery », a great number of sections are listed, the first four of which only correspond directly to your search; the following options concern general points on « arteries », but not in relation with «femoral artery ». Then, you will move back again to the sections dealing with «femoral artery ». Actually, these results concern all the items having a connection with the femoral artery and its branches. Each section is a hypertext on which the user clicks to view the relevant pages. On the other hand, we deplore that in the DVD Latin designations are not considered as headwords and are not consistently added to English designations.

Lastly, the visual menu bar located at the very top of the screen features legal information, instructions and an e-mail contact form to reach Thieme Verlag.

Overall, this is a wonderful browsing tool that is also most useful for a presentation including anatomical data (the copyright will be automatically assigned for each copied image).

It is highly recommended to all those who need to browse through general anatomical date or to use this book as a teaching tool.

No funds were received in support of this study.

Pierre Kehr Strasbourg

P. Kehr $(\square)$

Strasbourg, France

e-mail: kehrpier@aol.com 